\title{
Correlation Effects and Hidden Spin-Orbit Entangled Electronic Order in Parent and Electron-Doped Iridates $\mathrm{Sr}_{2} \mathrm{IrO}_{4}$
}

\author{
Sen Zhou, ${ }^{1}$ Kun Jiang, ${ }^{2}$ Hua Chen, ${ }^{3}$ and Ziqiang Wang ${ }^{2}$ \\ ${ }^{1}$ CAS Key Laboratory of Theoretical Physics, Institute of Theoretical Physics, \\ Chinese Academy of Sciences, Beijing 100190, China \\ and School of Physical Sciences, University of Chinese Academy of Sciences, Beijing 100049, China \\ ${ }^{2}$ Department of Physics, Boston College, Chestnut Hill, Massachusetts 02467, USA \\ ${ }^{3}$ International Center for Quantum Materials and School of Physics, Peking University, \\ Beijing 100871, China and Department of Physics, Zhejiang Normal University, Jinhua 321004, China \\ (Received 19 May 2017; revised manuscript received 11 August 2017; published 24 October 2017)
}

\begin{abstract}
Analogs of the high- $T_{c}$ cuprates have been long sought after in transition metal oxides. Because of the strong spin-orbit coupling, the $5 d$ perovskite iridates $\mathrm{Sr}_{2} \mathrm{IrO}_{4}$ exhibit a low-energy electronic structure remarkably similar to the cuprates. Whether a superconducting state exists as in the cuprates requires understanding the correlated spin-orbit entangled electronic states. Recent experiments discovered hidden order in the parent and electron-doped iridates, some with striking analogies to the cuprates, including Fermi surface pockets, Fermi arcs, and pseudogap. Here, we study the correlation and disorder effects in a five-orbital model derived from the band theory. We find that the experimental observations are consistent with a $d$-wave spin-orbit density wave order that breaks the symmetry of a joint twofold spin-orbital rotation followed by a lattice translation. There is a Berry phase and a plaquette spin flux due to spin procession as electrons hop between Ir atoms, akin to the intersite spin-orbit coupling in quantum spin Hall insulators. The associated staggered circulating $J_{\text {eff }}=1 / 2$ spin current can be probed by advanced techniques of spin-current detection in spintronics. This electronic order can emerge spontaneously from the intersite Coulomb interactions between the spatially extended iridium $5 d$ orbitals, turning the metallic state into an electron-doped quasi-2D Dirac semimetal with important implications on the possible superconducting state suggested by recent experiments.
\end{abstract}

DOI: 10.1103/PhysRevX.7.041018

$\mathrm{Sr}_{2} \mathrm{IrO}_{4}$ is isostructural to the cuprate $\mathrm{La}_{2} \mathrm{CuO}_{4}$ and becomes a canted antiferromagnetic (AFM) insulator below a Néel temperature $T_{N} \simeq 230 \mathrm{~K}[1,2]$. The canting of the inplane magnetic moments tracks the $\theta \simeq 11^{\circ}$ staggered $\mathrm{IrO}_{6}$ octahedra rotation about the $c$ axis [3-6] due to the strong spin-orbit coupling (SOC). The AFM insulating state arises from a novel interplay between SOC and electron correlation most easily understood near the atomic limit. $\operatorname{Ir}^{4+}$ has a $5 d^{5}$ configuration. The 5 electrons occupy the lower threefold $t_{2 g}$ orbitals separated from the higher twofold $e_{g}$ orbitals by the cubic crystal field $\Delta_{c}$. The strong atomic SOC $\lambda_{\text {SOC }}$ splits the $t_{2 g}$ orbitals into a low-lying $J_{\text {eff }}=3 / 2$ spin-orbit multiplet occupied by 4 electrons and a singly occupied $J_{\text {eff }}=1 / 2$ doublet. Assuming $\lambda_{\mathrm{SOC}}$ and $\Delta_{c}$ are sufficiently large compared to the relevant bandwidths when $\mathrm{Sr}_{2} \mathrm{IrO}_{4}$ crystalizes, a single $J_{\text {eff }}=1 / 2$ band is half filled and can be driven by a moderate local Coulomb repulsion $U$ to an AFM Mott

Published by the American Physical Society under the terms of the Creative Commons Attribution 4.0 International license. Further distribution of this work must maintain attribution to the author(s) and the published article's title, journal citation, and DOI.
Subject Areas: Condensed Matter Physics,

Strongly Correlated Materials insulating state $[1,2,7]$. The nature of the spin-orbit entangled insulating state has been studied using the localized picture based on the $J_{\text {eff }}=1 / 2$ pseudospin anisotropic Heisenberg model [7-11], the three-orbital Hubbard model for the $t_{2 g}$ electrons with SOC [12-15], and the microscopic correlated density functional theory such as the $\mathrm{LDA}+U$ and GGA $+U$ [1,16-18]. Moreover, carrier doping the AFM insulating state was proposed to potentially realize a $5 d t_{2 g}$-electron analog of the $3 d e_{g}$-electron high- $T_{c}$ cuprate superconductors $[8,12,13,19,20]$.

In this work, we study the hidden order in both stoichiometric and electron-doped $\mathrm{Sr}_{2} \mathrm{IrO}_{4}$ discovered recently by angle-resolved photoemission (ARPES) and scanning tunneling microscopy (STM). In high-quality undoped $\mathrm{Sr}_{2} \mathrm{IrO}_{4}$ samples, the most recent ARPES experiment [21] was able to resolve the broad spectra in the canted AFM insulator near the high-symmetry point $X=$ $(\pi, 0)$ and $(0, \pi)$ observed in earlier experiments [1,22-25] and reveal a degeneracy splitting of the quasiparticle (QP) dispersion, indicative of a symmetry-breaking hidden electronic order. Electron doping the AFM insulator has been achieved by La substitution $\left(\mathrm{Sr}_{2-x} \mathrm{La}_{x} \mathrm{IrO}_{4}\right)[21,26]$, oxygen deficiency $\left(\mathrm{Sr}_{2} \mathrm{IrO}_{4-\delta}\right)$ [27], and in situ potassium 
surface doping [28-30]. ARPES measurements showed that the collapse of the AFM insulating gap gives rise to a paramagnetic (PM) metallic state with Fermi surface (FS) pockets for bulk electron doping at $x=0.1$ [21] and to Fermi arcs [28] with $d$-wave-like pseudogaps around $X$ [21,29] under surface doping, in striking analogy to the high- $T_{c}$ cuprates. A hidden electronic order that breaks the rotation, inversion, and time-reversal symmetries has been observed in hole-doped $\mathrm{Sr}_{2} \mathrm{Ir}_{1-x} \mathrm{Rh}_{x} \mathrm{O}_{4}$ by optical second harmonic generation [31] and neutron scattering measurements [32]. However, similar experiments have not been performed in electron-doped iridates. Moreover, since the $\mathrm{Rh}$ substitution of the strongly spin-orbit coupled Ir in the Ir-O plane is very different than the electron doping by $\mathrm{La}$ substitution in the off-plane charge reservoir layers or surface $\mathrm{K}$ doping, we do not consider the hole-doped case further in this paper. Our focus is the hidden electronic order in the low-energy QP properties observed by ARPES and STM in undoped and electron-doped iridates.

To this end, we study the effects of correlation, SOC, and structure distortion on the spin-orbit entangled electronic states. We show that these remarkable QP properties can be described by a $d$-wave spin-orbit density wave ( $d$-SODW) with a circulating staggered $J_{\text {eff }}=1 / 2$ spin current that breaks the symmetry of twofold spin-orbital rotation followed by lattice translation. It gaps out the band touching point (BTP) at momentum $X$ and generates the electron pockets in the PM phase, and splits the degenerate band near $X$ in the canted AFM insulator, in remarkable agreement with experiments. We study the effects of disorder, which are shown to produce the pseudogap and Fermi arcs observed under surface doping. The electronic order induces a Berry phase associated with the staggered plaquette spin flux as electrons hop between the Ir sites via the oxygen due to spin precession. We argue that the hidden order has an electronic origin and can be generated spontaneously by the intersite Coulomb interactions due to the large spatial extent of the iridium $5 d$ orbitals, turning the metallic phase of the iridates into an electron-doped quasi-2D Dirac semimetal. These findings provide new insights and perspectives for understanding the possible emergence of a superconducting phase $[29,30]$.

We start with a realistic electronic structure calculation using the local-density approximation (LDA) including SOC and the structural distortion [33,34]. The result is shown in Fig. 1(a) for $\theta=11^{\circ}$. We then construct a two-dimensional tight-binding model including SOC $(\mathrm{TB}+\mathrm{SOC})$ for the low-energy band structure using five localized Wannier orbitals labeled by $\mu=1\left(d_{Y Z}\right), 2\left(d_{Z X}\right), 3\left(d_{X Y}\right), 4\left(d_{3 Z^{2}-R^{2}}\right)$, $5\left(d_{X^{2}-Y^{2}}\right)$. The TB + SOC Hamiltonian in the local coordinates that rotate with the octahedra is given by

$$
\begin{aligned}
\mathcal{H}_{0}= & \sum_{i j, \mu \nu, \sigma} t_{i j}^{\mu \nu, \sigma} d_{i \mu \sigma}^{\dagger} d_{j \nu \sigma}+\sum_{i \mu \sigma} \epsilon_{\mu} d_{i \mu \sigma}^{\dagger} d_{i \mu \sigma} \\
& +\sum_{i, \mu \nu, \sigma \sigma^{\prime}} \lambda_{\text {SOC }}\langle\mu|\mathbf{L}| \nu\rangle \cdot\left\langle\sigma|\mathbf{S}| \sigma^{\prime}\right\rangle d_{i \mu \sigma}^{\dagger} d_{i \nu \sigma^{\prime}} .
\end{aligned}
$$

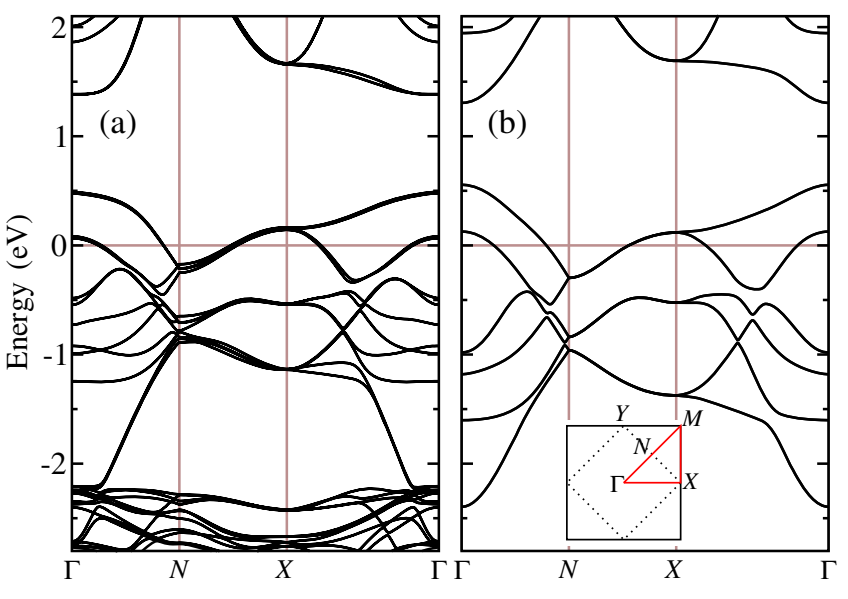

FIG. 1. Comparison of the band structures obtained using (a) LDA and (b) the five-orbital TB + SOC model with lattice distortion caused by staggered octahedra rotation. The doubling of bands and the small band splitting in (a) is due to the doubling of unit cell along the $c$ axis. The inset in (b) shows the one-Ir Brillouin zone (solid black lines), the reduced zone (dotted black lines), and the high-symmetry points labeled by $\Gamma=(0,0)$, $X=(\pi, 0), Y=(0, \pi), M=(\pi, \pi)$, and $N=(\pi / 2, \pi / 2)$.

Here, $d_{i \mu \sigma}^{\dagger}$ creates an electron with spin $\sigma$ in the $\mu$ th orbital at site $i$, and $t_{i j}^{\mu \nu, \sigma}$ is the spin-and-orbital-dependent complex hopping integrals between sites $i$ and $j$ of up to fifth nearest neighbors given in the Supplemental Material [35]. The second term in Eq. (1) denotes the crystalline electric field $(\mathrm{CEF}), \epsilon_{1, \ldots, 5}=(0,0,202,3054,3831) \mathrm{meV}$, with a separation of $\Delta_{c} \equiv 10 D q \approx 3.4 \mathrm{eV}$ between the $t_{2 g}$ and $e_{g}$ complexes. The last term in Eq. (1) is the atomic SOC with $\lambda_{\text {SOC }}=357 \mathrm{meV} ; \mathbf{S}$ and $\mathbf{L}$ are the spin and orbital angular momentum operators, respectively, whose matrix elements $S_{\sigma \sigma^{\prime}}^{\eta}=\left\langle\sigma\left|S^{\eta}\right| \sigma^{\prime}\right\rangle$, where $\eta=x, y, z$ in spin space and $L_{\mu \nu}^{\eta}=\left\langle\mu\left|L^{\eta}\right| \nu\right\rangle$ in the five $d$-orbital basis are given explicitly in the Supplemental Material [35]. The band dispersion produced by the $\mathrm{TB}+\mathrm{SOC}$ Hamiltonian is shown in Fig. 1(b), which captures faithfully the LDA band structure in Fig. 1(a) near the Fermi level. This is the first TB + SOC model of the first-principle electric structure of all five $d$ orbitals, which are necessary to describe quantitatively the lattice distortion and the atomic SOC. Zooming in to low energies in Fig. 2(a), it is clear that for the realistic bandwidths and CEF, the atomic SOC is insufficient to prevent two bands of predominantly $J_{\text {eff }}=1 / 2$ and $3 / 2$ characters to cross the Fermi level and give rise to two Fermi surfaces shown in Fig. 2(a).

To study the electron correlation effects, we consider the five-orbital Hubbard model $\mathcal{H}=\mathcal{H}_{0}+\mathcal{H}_{U}$ with

$$
\begin{aligned}
\mathcal{H}_{U}= & U \sum_{i, \mu} \hat{n}_{i \mu \uparrow} \hat{n}_{i \nu \downarrow}+\left(U^{\prime}-J / 2\right) \sum_{i, \mu<\nu} \hat{n}_{i \mu} \hat{n}_{i \nu} \\
& -J \sum_{i, \mu \neq \nu} \mathbf{S}_{i \mu} \cdot \mathbf{S}_{i \nu}+J \sum_{i, \mu \neq \nu} d_{i \mu \uparrow}^{\dagger} d_{i \mu \downarrow}^{\dagger} d_{i \nu \downarrow} d_{i \nu \uparrow},
\end{aligned}
$$



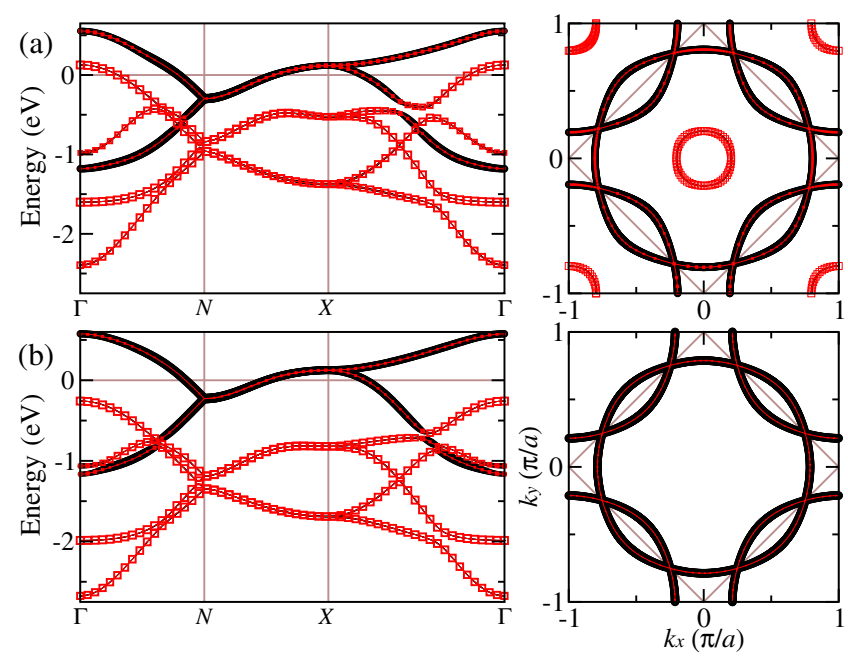

FIG. 2. The band dispersions and the corresponding FS of (a) the noninteracting $U=0$ and (b) the nonmagnetic state in the interacting $(U, J)=(1.2,0.05) \mathrm{eV}$ system at $x=0$. The thickness of the black lines denotes the content of the $J_{\text {eff }}=1 / 2$ doublet, while the size of the red open squares shows the content of the $J_{\text {eff }}=3 / 2$ multiplet.

where $U$ and $U^{\prime}$ are the local intraorbital and interorbital Coulomb repulsions and $J$ is the Hund's rule coupling with $U=U^{\prime}+2 J$. In the presence of SOC, the Hartree and exchange self-energies induced by $\mathcal{H}_{U}$ in the selfconsistent Hartree-Fock theory depend on the full spinorbital-dependent density matrix $n_{i \sigma \sigma^{\prime}}^{\mu \nu}=\left\langle d_{i \mu \sigma^{\prime}}^{\dagger} d_{i \nu \sigma^{\prime}}\right\rangle$. Local physical quantities in the ground state can be expressed in terms of $n_{\sigma \sigma^{\prime}}^{\mu \nu}$. The orbital occupation $n_{\mu}=\sum_{\sigma} n_{\sigma \sigma}^{\mu \mu}$, the spin density $S^{\eta}=\sum_{\mu, \sigma \sigma^{\prime}} S_{\sigma \sigma^{\prime}}^{\eta} n_{\sigma \sigma^{\prime}}^{\mu \mu}$, the orbital angular momentum $L^{\eta}=\sum_{\mu \neq \nu, \sigma} n_{\sigma \sigma}^{\mu \nu} L_{\mu \nu}^{\eta}$, and the $\operatorname{SOC} \Lambda^{\eta}=\sum_{\mu \nu, \sigma \sigma^{\prime}} n_{\sigma \sigma^{\prime}}^{\mu \nu} L_{\mu \nu}^{\eta} S_{\sigma \sigma^{\prime}}^{\eta}$ can be determined from the Hartree and exchange selfenergies.

Interacting electronic structure.-In the absence of symmetry breaking $(\langle\mathbf{S}\rangle=\langle\mathbf{L}\rangle=0)$, the only corrections to the electronic structure are the changes in the CEF and the renormalization of the atomic SOC. However, due to the cubic crystal field, $\Lambda^{\eta}$ are different along different directions. As a result, the correlation-induced SOC renormalization is both directional and orbital dependent, i.e., the $\lambda_{\text {SOC }}$ in Eq. (1) is replaced by $\lambda_{\mu \nu}^{\eta}=\lambda_{\text {SOC }}+\Delta \lambda_{\mu \nu}^{\eta}$, where $\Delta \lambda \propto \lambda_{\text {SOC }}\left(U^{\prime}-J\right) N_{F}$, where $N_{F}$ is the Fermi level density of states. In Fig. 2(b), the interacting electronic structure is shown at $(U, J)=(1.2,0.05) \mathrm{eV}$ and $x=0$. We find that the most important correlation effect on the electronic structure is the renormalization of $\lambda_{\text {SOC }}$, leading to a significantly enhanced effective SOC of $665 \mathrm{meV}$ for the $t_{2 g}$ complex much larger than the bare atomic value. As a consequence, the $J_{\text {eff }}=3 / 2$ band in the LDA band structure in Fig. 2(a) is pushed below the Fermi level together with the hole FS pockets around $\Gamma$ and $M$. As shown in Fig. 2(b), this gives rise to the single band crossing the Fermi level that is of dominant $J_{\text {eff }}=1 / 2$ character and folded by $(\pi, \pi)$ due to the lattice distortion. This correlation-induced band polarization through enhancement of the SOC by the Hubbard interaction enables the $J_{\text {eff }}=1 / 2$ picture.

Canted AFM insulator at $x=0$. - The fully selfconsistent Hartree-Fock ground state at nonzero values of $U$ and $J$ is indeed a canted AFM insulator as depicted in Fig. 3(a) with fully gapped QP dispersions shown in red solid lines in Fig. 3(b). The magnetization and the canting angle depend on the interaction parameters. For $(U, J)=(1.2,0.05) \mathrm{eV}$, the ordered magnetic moment is $|\langle\mathbf{L}\rangle+2\langle\mathbf{S}\rangle| \simeq 0.67 \mu_{B}$, with a canting angle of about $22^{\circ}$, which is larger than the experimental values. Note that weakcoupling approaches tend to overestimate magnetism; the results should be regarded as qualitative rather than quantitative [36], and our findings do not rely on these microscopic details. Comparing to the observed QP dispersion below the Fermi level in Fig. 3(b) reveals an important difference near the high-symmetry point $X$ : ARPES detects two split bands separated by about $200 \mathrm{meV}$, as shown by the superimposed blue squares. A symmetry analysis is necessary to understand this difference. In the PM state (black lines), there exists a BTP located at $X$ about $130 \mathrm{meV}$ above the Fermi level. This is a fourfold degenerate van Hove crossing point of the $J_{\text {eff }}=1 / 2$ doublet band with its $(\pi, \pi)$ folded counterpart by the structural distortion. The SOC does not affect the band degeneracy at $X$. Although the AFM order breaks the time-reversal $(\mathcal{T})$ symmetry and splits the Kramers pair, the QP band below the AFM gap in Fig. 3(b)
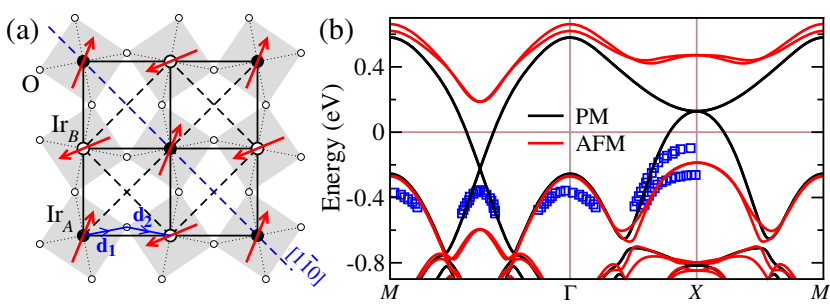

(c)
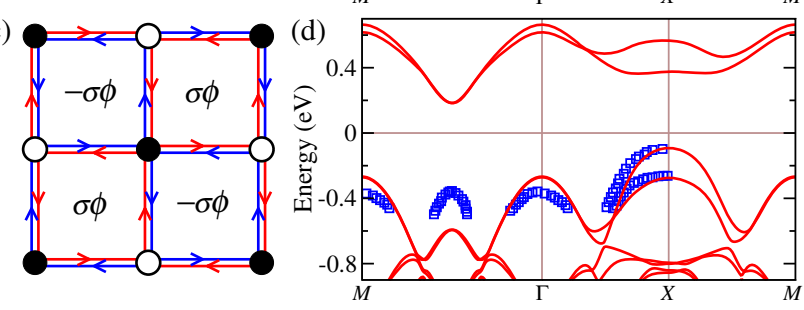

FIG. 3. (a) Schematics of the in-plane canted AFM moments on the Ir square lattice with a two-dimensional rendering of the staggered $\mathrm{IrO}_{6}$ octahedral rotation. (b) QP band dispersion in the canted AFM state (red solid lines) and the nonmagnetic state (black solid lines) at $(U, J)=(1.2,0.05) \mathrm{eV}$ and $x=0$. (c) Schematics of the $d$-wave $J_{\text {eff }}=1 / 2$ pseudospin current order and associated staggered pseudospin flux $\Phi_{\sigma}= \pm \sigma \phi$. (d) QP band dispersion in the canted AFM state with coexisting $d$-wave $J_{\text {eff }}=$ $1 / 2$ pseudospin current corresponding to $\Delta_{d}=30 \mathrm{meV}$. Open blue squares are data from ARPES experiments [21]. 
still maintains a twofold degeneracy. The two split bands observed by ARPES near $X$ are thus consistent with a $\pm 100 \mathrm{meV}$ band degeneracy lifting due to an additional symmetry breaking associated with a hidden order in the canted AFM state.

Symmetry analysis: Hidden order in AFM phase.-The symmetry implications on the electronic structure in $\mathrm{Sr}_{2} \mathrm{IrO}_{4}$ are subtle because of the strong SOC that renders the point group incomplete. In the absence of SOC, the doubly degenerate band in a two-sublattice collinear AFM state is related to a symmetry operation that flips the spin followed by an $A \leftrightarrow B$ sublattice translation $\tau_{A B}$ [37]. In the iridates, spin rotations are coupled to orbital spatial rotations due to the strong SOC. As a consequence, flipping the spin by the $180^{\circ}$ spin rotation $R_{s}^{\prime}(\pi)$ around the [11 10$]$ axis (direction of the canted FM moment) in Fig. 3(a) must be accompanied by a spatial $C_{2}^{\prime}$ rotation around the same axis, leading to the joint twofold rotation $\mathcal{J}_{2}^{\prime} \equiv R_{s}^{\prime}(\pi) \otimes C_{2}^{\prime}$. The corresponding operation in the spin-orbit entangled states must therefore be extended to $\mathcal{R}_{2}^{\prime} \equiv \mathcal{J}_{2}^{\prime} \otimes \tau_{A B}=R_{s}^{\prime}(\pi) \otimes C_{2}^{\prime} \otimes \tau_{A B} . \mathcal{R}_{2}^{\prime}$ is indeed a symmetry even in the presence of spin canting and the structural distortion. Thus, a hidden order that breaks $\mathcal{R}_{2}^{\prime}$ would lift the twofold band degeneracy. To determine the specific form of the hidden order, one can exhaust all possible interactions involving the low-energy $t_{2 g}$ orbitals that do not break lattice translation, identify those that break $\mathcal{R}_{2}^{\prime}$, and examine their momentum space anisotropy according to experiments [38]. Since the low-energy physics here is dominated by the $J_{\text {eff }}=1 / 2$ quantum states, the outcome can be suitably understood in the local pseudospin basis discussed in the Supplemental Material [35], $\left|J=1 / 2, J_{z}= \pm 1 / 2\right\rangle=\gamma_{ \pm}^{\dagger}|0\rangle$, where $\gamma_{\sigma}=$ $[1 /(\sqrt{3})]\left(i \sigma d_{Y Z, \bar{\sigma}}+d_{Z X, \bar{\sigma}}+i d_{X Y, \sigma}\right)$ creates the $J_{\text {eff }}=1 / 2$ doublet in QP excitations. Under the joint twofold spinorbital rotation $\mathcal{J}_{2}^{\prime \dagger} \sum_{\sigma} \sigma \gamma_{\sigma}^{\dagger} \gamma_{\sigma} \mathcal{J}_{2}^{\prime}=-\sum_{\sigma} \sigma \gamma_{\sigma}^{\dagger} \gamma_{\sigma}$; i.e., the pseudospin is flipped, as is its current. Considering the $C_{2}^{\prime}$ rotation and the sublattice translation $\tau_{A B}$, we arrive at the desired degeneracy lifting interaction,

$$
\mathcal{H}_{\Delta}=i \Delta_{d} \sum_{i \in A, \sigma} \sum_{j=i+\delta}(-1)^{i_{y}+j_{y}} \sigma \gamma_{i, \sigma}^{\dagger} \gamma_{j, \sigma}+\text { H.c., }
$$

where $\delta= \pm \hat{x}, \pm \hat{y}$ and $(-1)^{i_{y}+j_{y}}$ is the standard nearestneighbor (NN) $d$-wave form factor. $\mathcal{H}_{\Delta}$ maintains $\mathcal{T}$, but breaks $\mathcal{R}_{2}^{\prime}$, since $\mathcal{R}_{2}^{\prime \dagger} \mathcal{H}_{\Delta} \mathcal{R}_{2}^{\prime}=-\mathcal{H}_{\Delta}$. Equation (3) describes staggered ( $d$-wave) circulating $J_{\text {eff }}=1 / 2$ spin currents around each plaquette. Physically, the QPs traversing around a plaquette acquire a Berry phase from the enclosed spin flux for each pseudospin component in opposite directions, as shown in Fig. 3(c). A nonzero expectation value of $\mathcal{H}_{\Delta}$ in the ground state thus gives rise to the $J_{\text {eff }}=1 / 2 d$-wave pseudospin current order ( $d$-PSCO). Note that Eq. (3) can also be interpreted as a $J_{\text {eff }}=1 / 2$
$d$-SODW that can exhibit long-range order without the $\mathcal{T}$-breaking magnetic or charge current order. We use $d$-PSCO and $d$-SODW interchangeably. Moreover, $\mathcal{H}_{\Delta}$ also has the form of a $d$-wave spin-orbit coupling of the $J_{\text {eff }}=1 / 2$ QP and the $d$-wave form factor is crucial for breaking $\mathcal{R}_{2}^{\prime}$. Indeed, projecting the tight-binding $\mathcal{H}_{0}$ into the $J_{\text {eff }}=1 / 2$ basis in the local coordinates generates an extended $s$-wave SOC due to the structural distortion [19], which is invariant under $\mathcal{R}_{2}^{\prime}$ and already present in the band theory.

Including $\mathcal{H}_{\Delta}$ in the total Hamiltonian $\mathcal{H}=\mathcal{H}_{0}+\mathcal{H}_{U}+$ $\mathcal{H}_{\Delta}$, the canted AFM phase at $x=0$ indeed coexists with $d$-PSCO for remarkably small $\Delta_{d}$. The calculated QP dispersion is shown in Fig. 3(d) for $\Delta_{d}=30 \mathrm{meV}$, which produces a $\pm 100 \mathrm{meV}$ degeneracy splitting at $X$ in remarkable agreement with experiments [21]. The NN $J_{\text {eff }}=1 / 2 \quad$ QP correlator $\chi_{i j}^{\sigma}=\left\langle\gamma_{i \sigma}^{\dagger} \gamma_{j \sigma}\right\rangle=\chi_{i j}^{\prime}+i \sigma \chi_{i j}^{\prime \prime}$, and the staggered pseudospin flux $\Phi_{\sigma}= \pm \sigma \phi, \quad \phi=$ $\sum_{\square} \tan ^{-1}\left(\chi_{i j}^{\prime \prime} / \chi_{i j}^{\prime}\right) \simeq 0.055 \pi$. The QP number current on a link is $J_{i j}^{\sigma} \propto \operatorname{Im} \chi_{i j}^{\sigma}$, such that the pseudospin current $J_{i j}^{\mathrm{ps}}=\sum_{\sigma} \sigma J_{i j}^{\sigma} \neq 0$, whereas the charge current $J_{i j}=\sum_{\sigma} J_{i j}^{\sigma}=0$, giving rise to the novel $\mathcal{T}$-symmetric $d$-SODW state with $d$-wave pseudospin current. The $d$ wave form factor $\left(\cos k_{x}-\cos k_{y}\right)$ ensures splitting is largest at $X$, and vanishes along the $\Gamma-N-M$ path in the BZ in agreement with the superimposed ARPES data. The mismatch in the band positions near the $N$ point is because the band crossing in the PM phase [black lines in Fig. 3(b)] is too far below the Fermi level in the weakcoupling theory. The correlation-induced band narrowing in the strong-coupling treatment of $U$, such as in the Gutzwiller approximation or the slave boson approaches, would significantly reduce this quantitative discrepancy.

A closer look at the calculated band dispersion near $X$ in Fig. 3(d) reveals a weak asymmetry between the directions $\Gamma \rightarrow X$ and $M \rightarrow X$ (equivalent to $\Gamma \rightarrow Y$ in 2D) related by $90^{\circ}$ rotations. In the absence of structure distortion, although the magnetic ordered state and the $J_{\text {eff }}=1 / 2$ $d$-PSCO break the fourfold rotation symmetry $\left(C_{4}\right.$ plus $90^{\circ}$ spin rotation due to SOC), nematic asymmetry in the electronic dispersion should be absent since $\mathcal{J}_{2}^{\prime} \otimes \mathcal{T}$ remains a good symmetry that effectively interchanges $k_{x} \leftrightarrow k_{y}$. Thus, the weak asymmetry in the QP dispersions near $X$ is due to the structure distortion that breaks $\mathcal{J}_{2}^{\prime} \otimes \mathcal{T}$, causing a mixing of the $s$-wave SOC with the $d$-wave pseudospin current on the bonds. Direct measurement of the QP dispersion along $\Gamma \rightarrow Y$ by ARPES would be very desirable for comparison in Fig. 3(d) in order to verify the weak nematicity induced by the proposed $d$-PSCO.

The ability of the $d$-PSCO to split the band degeneracy around $X$ offers insights into the effects of disorder. A significant source of disorder that affects the sample quality even in the undoped $\mathrm{Sr}_{2} \mathrm{IrO}_{4}$ is the disorder in the structural distortion, in particular, the spatially inhomogeneous 
variations in the staggered $\mathrm{IrO}_{6}$ octahedral rotation and thus those in the $s$-wave SOC for the $J_{\text {eff }}=1 / 2$ QP [19] about the average value. This necessarily generates a local distribution of the $d$-PSCO with "smeared out" band splittings that contribute to the broad spectrum near $X$ observed in all ARPES measurement [1,21-25]. The band splitting was resolved recently in presumably better quality samples [21].

Electron-doped PM state.-It is remarkable that such a novel spin-orbit entangled order in the canted AFM phase can also account for the Fermi pocket, Fermi arc, and pseudogap phenomena in the PM phase following the collapse of the AFM insulating gap in electron-doped $\mathrm{Sr}_{2} \mathrm{IrO}_{4}$. Indeed, we could have started the discussion with the electron-doped case, as we show below, and arrived at the same conclusion for the $d$-wave SODW order. More detailed symmetry analysis is given in Sec. D of the Supplemental Material [35].

The calculated QP band dispersions in the PM state without $\mathcal{H}_{\Delta}$ are shown in Fig. 4(a) for $(U, J)=(1.4,0) \mathrm{eV}$ at $x=0.1$. Despite the absence of AFM order, the bands are still folded by $(\pi, \pi)$ due to the staggered $\mathrm{IrO}_{6}$ octahedra rotation. Moreover, electron doping has moved the Fermi energy $\left(E_{F}\right)$ upward to within $70 \mathrm{meV}$ of the BTP at $X$ in Fig. 4(a). Comparing to the superimposed dispersion measured by ARPES [21], the electron FS pocket around $(\pi / 2, \pi / 2)$ in Fig. 4(b) is indeed observed with the QP dispersion extending from $E_{F}$ down to and beyond the "Dirac crossing," consistent with the calculated QP dispersion in Fig. 4(a). However, the hole FS pocket around $X$ in Fig. 4(b) was not observed by ARPES; the measured QP peak near $X$ follows the band dispersion below $E_{F}$ but loses its intensity before reaching the
Fermi level, leading to the emergence of a $\sim 30 \mathrm{meV}$ gap at the $X$ point [21]. It is natural to suspect that short-range AFM order or fluctuations $[39,40]$ may be responsible for the (pseudo)gap behavior [41]. However, the latter would produce a significant energy gap in the QP dispersion around $(\pi / 2, \pi / 2)$ as well, which was not detected by ARPES [21].

We propose that closing the AFM gap by electron doping reveals the $d$-PSCO already present in the canted AFM phase. A gap opening at $X$ requires symmetry breaking and lifting of the degeneracy at the BTP. The $\mathcal{T}$ symmetry in the PM phase protects the twofold Kramers degeneracy. However, the proximity of $E_{F}$ to the van Hove BTP increases the propensity toward $d$-PSCO that spontaneously breaks the $\mathcal{R}_{2}^{\prime}$ symmetry and lifts the remaining twofold degeneracy by gapping out the BTP. Figure 4(c) shows the calculated QP dispersion in the presence of $\mathcal{H}_{\Delta}$ in Eq. (3) with an identical magnitude $\Delta_{d}=30 \mathrm{meV}$ used in the AFM phase at $x=0$. The induced staggered pseudospin flux is $\pm \sigma \phi$ with $\phi=0.589 \pi$. The $d$-PSCO splits the BTP by about $200 \mathrm{meV}$ and produces a 30-meV gap in the QP dispersion at $X$ shown in Fig. 4(c), leaving behind only the electron pocket around $(\pi / 2, \pi / 2)$ in Fig. 4(d) occupied by the doped carriers, in very good agreement with the superimposed ARPES data in electron-doped $\mathrm{Sr}_{2-x} \mathrm{La}_{x} \mathrm{IrO}_{4}$ at $x=0.1$ [21]. As in the undoped case, it would be very desirable to have the measured dispersions available along $\Gamma \rightarrow Y$ for comparison to the predicted weak nematicity and along $X \rightarrow N$ for the existence of the Dirac point. Figure 4(d) shows that the QP spectral weight is much larger on the outer half than on the inner half of the FS pockets, consistent with the former being the main $J_{\text {eff }}=1 / 2$ QP band while the latter the folded band [Fig. 4(c)] by the structural distortion
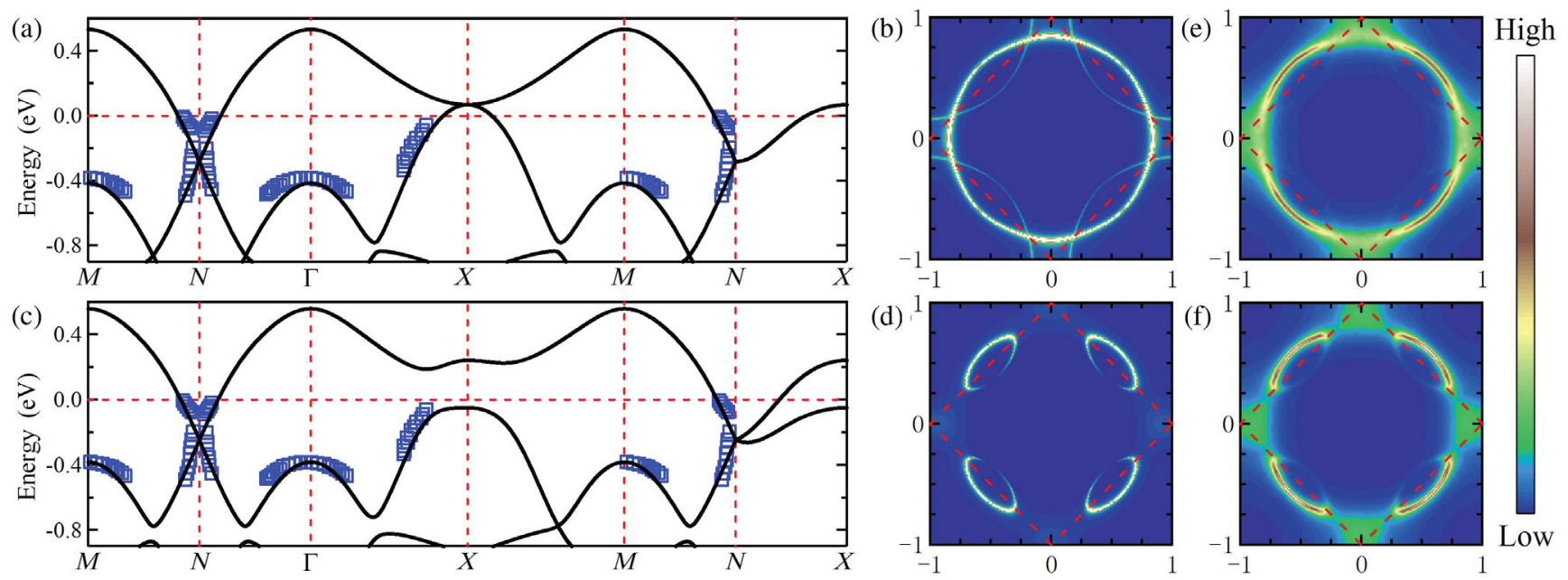

FIG. 4. The PM phase at $x=0.1$ and $(U, J)=(1.4,0) \mathrm{eV}$. (a),(b) Band dispersion in the absence of $H_{\Delta}$, i.e., without $d$-PSCO (black lines) and the spectral intensity of the corresponding FS. (c),(d) Band dispersion in the presence of $d$-PSCO for $\Delta_{d}=30$ meV showing the gapping of the BTP at $X$ (red lines) and the spectral intensity of the corresponding FS. (e) FS spectrum in the presence of disorder with $\Delta_{d}=0$ and $\varepsilon=60 \mathrm{meV}$, showing the Fermi arcs. (f) FS spectrum in the disordered $d$-PSCO with $\Delta_{d}=30 \mathrm{meV}$ and $\varepsilon=60 \mathrm{meV}$. ARPES data [21] are superimposed as open blue squares. 
and $d$-SODW order. Significant photon-energy-dependent spectral weight anisotropy on the electron pocket has been observed by ARPES. The current theory, however, cannot explain the surprising result that, at certain photon energies, the folded portion has higher intensity, which may be caused by the matrix element effects [21].

Disorder effects, Fermi arc, and pseudogap behavior.We explain that the native disorder in the structural distortion of the undoped iridates leads to local modulations of the $d$-PSCO and the broadening of the spectral function near $X$ in the canted AFM state. Career doping usually introduces additional sources of disorder. In the high- $T_{c}$ cuprates, doping-induced disorder contributes significantly to the electronic inhomogeneity [42-46]. Moreover, when a system sits close to a long-range electronic order, disorder can pin the low-energy quantum fluctuations to form a spatially inhomogeneous state with glassy or short-range order that inherits certain spectroscopic properties of the ordered state. The $d$-wave valence bond glass is such an example proposed for the pseudogap phase with Fermi arcs in underdoped cuprates [47]. To model the disorder effects, we rewrite Eq. (3) in real space,

$$
\mathcal{H}_{\Delta}^{\mathrm{dis}}=i \sum_{i \in A, \sigma} \sum_{j=i+\delta} \Delta_{i j} \sigma \gamma_{i, \sigma}^{\dagger} \gamma_{j, \sigma}+\text { H.c. }
$$

where the bond coupling $\Delta_{i j}=(-1)^{i_{y}+j_{y}} \Delta_{d}+\delta \Delta_{i j}$ contains an average $d$-wave contribution $\Delta_{d}$ and a random $\delta \Delta_{i j}$ taken from a Gaussian distribution of zero mean and standard deviation $\varepsilon$. Note that the disordered $\delta \Delta_{i j}$ necessarily involve spatially fluctuating $J_{\text {eff }}=1 / 2 d$-wave and $s$-wave SODW or spin currents. We first set $\Delta_{d}=0$, such that the $d$-PSCO vanishes on average $\left\langle\mathcal{H}_{\Delta}\right\rangle=0$, i.e., without long-range order, but its moment $\left\langle\mathcal{H}_{\Delta}^{2}\right\rangle \neq 0$, giving rise to short-range ordered SODW or equivalently a valence bond glass of $J_{\text {eff }}=1 / 2$ spin current. The FS obtained with quenched disorder average [48] is plotted in Fig. 4(e) at $x=0.1$ for $\varepsilon=60 \mathrm{meV}$, which shows the destruction of the FS sections around $X$ as in the clean case with long-range $d$-PSCO. More remarkable is the emergence of the fullfledged Fermi arcs as the folded part of the FS is destroyed by the scattering due to spatially fluctuating pseudospin current, in good agreement with the observed Fermi arcs and $d$-wave-like pseudogaps by ARPES and STM in heavily surface-K-doped iridates [28-30]. Figure 4(f) is a spectral intensity plot of the FS when the disordered pseudospin currents fluctuate spatially around a nonzero mean of the $d$-PSCO with $\Delta_{d}= \pm 30 \mathrm{meV}$ to account for averaging over two domains. Since the static order is comparable to the disorder strength, although the spectral weight on the inner halves of the electron pockets is suppressed, the bending over of the Fermi arcs remains visible.

Discussions.-We show that the highly unconventional QP properties observed in both the parent and electrondoped square lattice iridates can be described by the same $d$-wave spin current or SODW order proposed in Eq. (3). The basic mechanism is that the spin-orbit entangled electronic order breaks the "hidden" $R_{2}^{\prime}$ symmetry, but it is equally surprising and reassuring that the manifestations of the corresponding degeneracy lifting of the quantum states at the high-symmetry point $X$ can account for the ARPES and STM observations in both the electron-doped PM pseudogap phase as well as the undoped canted AFM insulator. Other symmetry-breaking interactions capable of lifting the degeneracy at $X$, such as those discussed in Sec. D of the Supplemental Material [35], do not have this property. For example, the $\mathcal{T}$-breaking $d$-wave circulating current or the staggered flux order gaps out the BTP at $X$ in the PM phase, but cannot produce the band splitting in the AFM insulator, whereas the $d$-wave spin nematic order produces the band splitting in the canted AFM insulator, but cannot remove the hole Fermi surface pocket around $X$ in the electron-doped PM phase. The $d$-wave bond nematic order does not split the degeneracy in the AFM insulator and produces strongly nematic QP band dispersion near $X$ in the PM metallic state, incompatible with experimental findings. It is important to note that the proposed $d$-PSCO in Eq. (3) is time-reversal invariant. Thus it does not describe the time-reversal breaking hidden order in holedoped $\mathrm{Sr}_{2} \mathrm{Ir}_{1-x} \mathrm{Rh}_{x} \mathrm{O}_{4}$ observed by SHG [31] and neutron scattering measurements [32]. Given the different nature of the chemical doping and the extension of the magnetically ordered phase, it is very desirable for these measurements to be carried out for the electron-doped iridates.

The most direct manifestation of the $d$-PSCO order is the Fermi pocket and the Fermi arc or pseudogap behavior in the electron-doped PM phase. However, since it breaks different symmetries than the canted AFM order, two separate phase transitions are expected in the undoped and lightly electron-doped AFM phase, which should be observable with improved sample quality. Still, direct experimental detection of the $d$-PSCO would be most convincing. The circulating spin current can in principle be probed experimentally by the recent advances in spin current detection in spintronics using optical SHG [49] and $\mathrm{x}$-ray magnetic circular dichroism [50]. However, the $d$-wave or the staggered nature of the ordered current makes the detection of a "net" spin current or spin flux by these techniques challenging. Hence, utilizing the response of the $d$-PSCO to the local environment near nonmagnetic or magnetic impurities [51,52] may be more suitable for its detection by local probes such as NMR and STM, in addition to the abovementioned methods. It may be possible to detect the $d$-wave form factor from the spatial patterns of the local density of states accessible by STM. A distribution of net spin flux or current may also emerge and be picked up by spintronic techniques near nonmagnetic impurities, or even net charge flux or current near magnetic impurities that can be detected by local magnetometry, such as an atomic force magnetometer. 
The undoped $\mathrm{Sr}_{2} \mathrm{IrO}_{4}$ does exhibit a lowering of crystal symmetry at high temperatures observed by neutron and resonant X-ray scattering [53-55] and shown by optical SHG as due to the staggered tetragonal distortion of the $\mathrm{IrO}_{6}$ octahedra [56]. However, the important $d$-wave factor in $H_{\Delta}$ requires additional $C_{4}$ symmetry breaking. To illustrate this point, it is instructive to consider the spin precession due to SOC when the QP hops between the NN Ir atoms via the oxygen, as indicated by the vectors $\vec{d}_{1}$ and $\vec{d}_{2}$ in Fig. 3(a). This intersite SOC [57] is given by $i \lambda_{12}\left(\vec{d}_{1} \times \vec{d}_{2}\right)$. $\vec{\tau}_{\sigma \sigma^{\prime}} \gamma_{1 \sigma}^{\dagger} \gamma_{2 \sigma^{\prime}}$, which leads to Eq. (3) if $C_{4}$ symmetry is broken. Since in the undoped canted AFM state the SHG signals even break the $C_{2}$ symmetry [31], the two-dimensional $d$ PSCO we propose here is allowed although it does not break inversion or $C_{2}$ within a single layer, nor does it break $\mathcal{T}$ already broken by magnetic order. Further studies on the $c$ axis stacking of the $d$-PSCO and the magnetic order [58] are necessary in order to compare directly to the nonlinear optics and the interpretation in terms of intracell loop currents [31].

It is likely that the main driving force behind the $d$-PSCO has an electronic origin. The nature of the nonlocal spin current suggests that it may emerge from intersite electronic interactions. Since the $5 d$ orbitals have a large spatial extent, the NN interatomic Coulomb interaction $V$ can be important. Indeed, Eq. (3) can be obtained by decoupling $V$ between the $J_{\text {eff }}=1 / 2$ QPs, as in the study of topological Mott insulators [59]. Our preliminary calculations using a single-band $t-U-V$ model for the $J_{\text {eff }}=1 / 2$ QPs, with band parameters extracted from the present theory, indeed show spontaneous generation of the $d$-PSCO above a critical $V$ both in the PM phase and in the AFM phase coexisting and competing with the AFM order. The nonlocal charge fluctuations of spin-orbit coupled QPs governed by the interatomic $V$, unfavorable for $d$-wave pairing via spin fluctuations or the superexchange interaction, present a crucial difference between the iridates and the cuprates.

The $d$-PSCO offers a new perspective on the electronic structure of the iridates since it splits the QP band degeneracy from $X$ to $N$ except for the Dirac point at $(\pi / 2, \pi / 2)$ [see Fig. 4(c)] protected by the nonsymmorphic space group symmetries [60]. Thus, the metallic state of the iridates behaves as an electron-doped quasi-2D Dirac semimetal, which may play an essential role for studying electronic pairing and the possible emergence of superconductivity in $\mathrm{Sr}_{2-x} \mathrm{La}_{x} \mathrm{IrO}_{4}[29,30]$.

We thank Stephen Wilson, Xi Dai, and Jiadong Zang for useful discussions. This work is supported by the U.S. Department of Energy, Basic Energy Sciences Grant No. DE-FG02-99ER45747 (Z. W. and K. J.) and the Key Research Program of Frontier Sciences, CAS, Grant No. QYZDB-SSW-SYS012 (S. Z.). Numerical calculations were performed on HPC Cluster of ITP-CAS. Z. W. thanks the hospitality of Aspen Center for Physics and the support of ACP NSF Grant No. PHY-1066293.
[1] B. J. Kim et al., Novel $J_{\text {eff }}=1 / 2$ Mott State Induced by Relativistic Spin-Orbit Coupling in $\mathrm{Sr}_{2} \mathrm{IrO}_{4}$, Phys. Rev. Lett. 101, 076402 (2008).

[2] B. J. Kim, H. Ohsumi, T. Komesu, S. Sakai, T. Morita, H. Takagi, and T. Arima, Phase-Sensitive Observation of a Spin-Orbital Mott State in $\mathrm{Sr}_{2} \mathrm{IrO}_{4}$, Science 323, 1329 (2009).

[3] Q. Huang, J.L. Soubeyroux, O. Chmaissem, I.Natali Sora, A. Santoro, R.J. Cava, J.J. Krajewski, and W.F. Peck, Neutron Powder Diffraction Study of the Crystal Structures of $\mathrm{Sr}_{2} \mathrm{RuO}_{4}$ and $\mathrm{Sr}_{2} \mathrm{IrO}_{4}$ at Room Temperature and at $10 \mathrm{~K}$, J. Solid State Chem. 112, 355 (1994).

[4] R. J. Cava, B. Batlogg, K. Kiyono, H. Takagi, J. J. Krajewski, W. F. Peck, L. W. Rupp, and C. H. Chen, Localized-toItinerant Electron Transition in $\mathrm{Sr}_{2} \mathrm{Ir}_{1-x} \mathrm{Ru}_{x} \mathrm{O}_{4}$, Phys. Rev. B 49, 11890 (1994).

[5] G. Cao, J. Bolivar, S. McCall, J. E. Crow, and R. P. Guertin, Weak Ferromagnetism, Metal-to-Nonmetal Transition, and Negative Differential Resistivity in Single-Crystal $\mathrm{Sr}_{2} \mathrm{IrO}_{4}$, Phys. Rev. B 57, R11039 (1998).

[6] M. K. Crawford, M. A. Subramanian, R. L. Harlow, J. A. Fernandez-Baca, Z. R. Wang, and D. C. Johnston, Structural and Magnetic Studies of $\mathrm{Sr}_{2} \mathrm{IrO}_{4}$, Phys. Rev. B 49, 9198 (1994).

[7] G. Jackeli and G. Khaliullin, Mott Insulators in the Strong Spin-Orbit Coupling Limit: From Heisenberg to a Quantum Compass and Kitaev Models, Phys. Rev. Lett. 102, 017205 (2009).

[8] J. W. Kim, Y. Choi, Jungho Kim, J. F. Mitchell, G. Jackeli, M. Daghofer, J. van den Brink, G. Khaliullin, and B. J. Kim, Dimensionality Driven Spin-Flop Transition in Layered Iridates, Phys. Rev. Lett. 109, 037204 (2012).

[9] S. Fujiyama, H. Ohsumi, T. Komesu, J. Matsuno, B. J. Kim, M. Takata, T. Arima, and H. Takagi, Two-Dimensional Heisenberg Behavior of $J_{\text {eff }}=1 / 2$ Isospins in the Paramagnetic State of the Spin-Orbital Mott Insulator $\mathrm{Sr}_{2} \mathrm{IrO}_{4}$, Phys. Rev. Lett. 108, 247212 (2012).

[10] N. B. Perkins, Y. Sizyuk, and P. Wölfle, Interplay of ManyBody and Single-Particle Interactions in Iridates and Rhodates, Phys. Rev. B 89, 035143 (2014).

[11] J.-M. Carter, V. Shankar V., and H.-Y. Kee, Theory of Metal-Insulator Transition in the Family of Perovskite Iridum Oxides, Phys. Rev. B 88, 035111 (2013).

[12] H. Watanabe, T. Shirakawa, and S. Yunoki, Microscopic Study of a Spin-Orbit-Induced Mott Insulator in Ir Oxides, Phys. Rev. Lett. 105, 216410 (2010).

[13] H. Watanabe, T. Shirakawa, and S. Yunoki, Theoretical Study of Insulating Mechanism in Multiorbital Hubbard Models with a Large Spin-Orbit Coupling: Slater versus Mott Scenario in $\mathrm{Sr}_{2} \mathrm{IrO}_{4}$, Phys. Rev. B 89, 165115 (2014).

[14] R. Arita, J. Kuneš, A. V. Kozhevnikov, A. G. Eguiluz, and M. Imada, Ab Initio Studies on the Interplay between SpinOrbit Interaction and Coulomb Correlation in $\mathrm{Sr}_{2} \mathrm{IrO}_{4}$ and $\mathrm{Ba}_{2} \mathrm{IrO}_{4}$, Phys. Rev. Lett. 108, 086403 (2012).

[15] D. Hsieh, F. Mahmood, D. H. Torchinsky, G. Cao, and N. Gedik, Observation of a Metal-to-Insulator Transition with Both Mott-Hubbard and Slater Characteristics in $\mathrm{Sr}_{2} \mathrm{IrO}_{4}$ from Time-Resolved Photocarrier Dynamics, Phys. Rev. B 86, 035128 (2012). 
[16] H. Jin, H. Jeong, T. Ozaki, and J.-J. Yu, Anisotropic Exchange Interactions of Spin-Orbit-Integrated States in $\mathrm{Sr}_{2} \mathrm{IrO}_{4}$, Phys. Rev. B 80, 075112 (2009).

[17] P. Liu, S. Khmelevskyi, B. Kim, M. Marsman, D. Li, X.-Q. Chen, D. D. Sarma, G. Kresse, and C. Franchini, Anisotropic Magnetic Couplings and Structure-Driven Canted to Collinear Transitions in $\mathrm{Sr}_{2} \mathrm{IrO}_{4}$ by Magnetically Constrained noncollinear DFT, Phys. Rev. B 92, 054428 (2015).

[18] P. Liu, M. Reticcioli, B. Kim, A. Continenza, G. Kresse, D. D. Sarma, X.-Q. Chen, and C. Franchini, Electron and Hole Doping in the Relativistic Mott Insulator $\mathrm{Sr}_{2} \mathrm{IrO}_{4}$ : A FirstPrinciples Study Using Band Unfolding Technique, Phys. Rev. B 94, 195145 (2016).

[19] Fa Wang and T. Senthil, Twisted Hubbard Model for $\mathrm{Sr}_{2} \mathrm{IrO}_{4}$ : Magnetism and Possible High Temperature Superconductivity, Phys. Rev. Lett. 106136402 (2011).

[20] Z.-Y. Meng, Y. B. Kim, and H.-Y. Kee, Odd-Parity Triplet Superconducting Phase in Multiorbital Materials with a Strong Spin-Orbit Coupling: Application to Doped $\mathrm{Sr}_{2} \mathrm{IrO}_{4}$, Phys. Rev. Lett. 113, 177003 (2014).

[21] A. de la Torre et al., Collapse of the Mott Gap and Emergence of a Nodal Liquid in Lightly Doped $\mathrm{Sr}_{2} \mathrm{IrO}_{4}$, Phys. Rev. Lett. 115, 176402 (2015).

[22] S. Moser et al., The Electronic Structure of the HighSymmetry Perovskite Iridate $\mathrm{Ba}_{2} \mathrm{IrO}_{4}$, New J. Phys. 16, 013008 (2014).

[23] M. Uchida, Y. F. Nie, P. D. C. King, C. H. Kim, C. J. Fennie, D. G. Schlom, and K. M. Shen, Correlated vs. Conventional Insulating Behavior in the $\mathrm{J}_{\mathrm{eff}}=\frac{1}{2}$ vs. $\frac{3}{2}$ Bands in the Layered Iridate $\mathrm{Ba}_{2} \mathrm{IrO}_{4}$, Phys. Rev. B 90, 075142 (2014).

[24] V. Brouet et al., Transfer of Spectral Weight across the Gap of $\mathrm{Sr}_{2} \mathrm{IrO}_{4}$ Induced by La Doping, Phys. Rev. B 92, 081117 (R) (2015).

[25] Y.F. Nie et al., Interplay of Spin-Orbit Interactions, Dimensionality, and Octahedral Rotations in Semimetallic $\mathrm{SrIrO}_{3}$, Phys. Rev. Lett. 114, 016401 (2015).

[26] M.-Y. Li, Z.-T. Liu, H.-F. Yang, J.-L. Zhao, Q. Yao, C.-Cong Fan, J.-S. Liu, B. Gao, D.-W. Shen, and X.-M. Xie, Tuning the Electronic Structure of Thin Films by Bulk Electronic Doping Using Molecular Beam Epitaxy, Chin. Phys. Lett. 32, 057402 (2015).

[27] O. B. Korneta, T. Qi, S. Chikara, S. Parkin, L. E. De Long, P. Schlottmann, and G. Cao, Electron-Doped $\mathrm{Sr}_{2} \mathrm{IrO}_{4-\delta}(0 \leq \delta \leq 0.04)$ : Evolution of a Disordered $J_{\mathrm{eff}}=$ $\frac{1}{2}$ Mott Insulator into an Exotic Metallic State, Phys. Rev. B 82, 115117 (2010).

[28] Y. K. Kim, O. Krupin, J. D. Denlinger, A. Bostwick, E. Rotenberg, Q. Zhao, J. F. Mitchell, J. W. Allen, and B. J. Kim, Fermi Arcs in a Doped Pseudospin-1/2 Heisenberg Antiferromagnet, Science 345, 187 (2014).

[29] Y. K. Kim, N. H. Sung, J. D. Denlinger, and B. J. Kim, Observation of a d-Wave Gap in Electron-Doped $\mathrm{Sr}_{2} \mathrm{IrO}_{4}$, Nat. Phys. 12, 37 (2016).

[30] Y. J. Yan, M. Q. Ren, H. C. Xu, B. P. Xie, R. Tao, H. Y. Choi, N. Lee, Y. J. Choi, T. Zhang, and D. L. Feng, Electron-Doped $\mathrm{Sr}_{2} \mathrm{IrO}_{4}$ : An Analogue of Hole-Doped Cuprate Superconductors Demonstrated by Scanning Tunneling Microscopy, Phys. Rev. X 5, 041018 (2015).
[31] L. Zhao, D. H. Torchinsky, H. Chu, V. Ivanov, R. Lifshitz, R. Flint, T. Qi, G. Cao, and D. Hsieh, Evidence of an Odd-Parity Hidden Order in a Spin-Orbit Coupled Correlated Iridate, Nat. Phys. 12, 32 (2016).

[32] J. Jeong, Y. Sidis, A. Louat, V. Brouet, and P. Bourges, Time-Reversal Symmetry Breaking Hidden Order in $\mathrm{Sr}_{2}(\mathrm{Ir}, \mathrm{Rh}) \mathrm{O}_{4}$, Nat. Commun. 8, 15119 (2017).

[33] C. Martins, M. Aichhorn, L. Vaugier, and S. Biermann, Reduced Effective Spin-Orbital Degeneracy and Spin-Orbital Ordering in Paramagnetic Transition-Metal Oxides: $\mathrm{Sr}_{2} \mathrm{IrO}_{4}$ versus $\mathrm{Sr}_{2} \mathrm{RhO}_{4}$, Phys. Rev. Lett. 107, 266404 (2011).

[34] P. Giannozzi et al., QUANTUM ESPRESSO: A Modular and Open-Source Software Project for Quantum Simulations of Materials, J. Phys. Condens. Matter 21, 395502 (2009).

[35] See Supplemental Material at http://link.aps.org/ supplemental/10.1103/PhysRevX.7.041018 for more detailed discussions on (a) Orbital angular momentum and spin orbit coupling, (b) LDA band structure and TB + SOC model, (c) $J_{\text {eff }}=1 / 2$ doublet, and (d) Symmetry and symmetry breaking interactions.

[36] We have checked that using the value of $11^{\circ}$ for the canting angle, which is close to the measured value, does not give any appreciable changes in the obtained band dispersion.

[37] E. Berg, C.-C. Chen, and S. A. Kivelson, Stability of Nodal Quasiparticles in Superconductors with Coexisting Orders, Phys. Rev. Lett. 100, 027003 (2008).

[38] K. Jiang, J.-P. Hu, H. Ding, and Z. Wang, Interatomic Coulomb Interaction and Electron Nematic Bond Order in FeSe, Phys. Rev. B 93, 115138 (2016).

[39] X. Liu et al., Anisotropic Softening of Magnetic Excitations in Lightly Electron-Doped $\mathrm{Sr}_{2} \mathrm{IrO}_{4}$, Phys. Rev. B 93, 241102(R) (2016).

[40] H. Gretarsson et al., Persistent Paramagnons Deep in the Metallic Phase of $\mathrm{Sr}_{2-x} \mathrm{La}_{x} \mathrm{IrO}_{4}$, Phys. Rev. Lett. 117, 107001 (2016).

[41] Hu Wang, Shun-Li Yu, and Jian-Xin Li, Fermi Arcs, Pseudogap, and Collective Excitations in Doped $\mathrm{Sr}_{2} \mathrm{IrO}_{4}$ : A Generalized Fluctuation Exchange Study, Phys. Rev. B 91, 165138 (2015).

[42] S. H. Pan et al., Microscopic Electronic Inhomogeneity in the High- $T_{c}$ Superconductor $\mathrm{Bi}_{2} \mathrm{Sr}_{2} \mathrm{CaCu}_{2} \mathrm{O}_{8+x}$, Nature (London) 413, 282 (2001).

[43] Z. Wang, J. R. Engelbrecht, S. C. Wang, H. Ding, and S. H. Pan, Inhomogeneous $d$-Wave Superconducting State of a Doped Mott Insulator, Phys. Rev. B 65, 064509 (2002).

[44] K. McElroy et al., Atomic-Scale Sources and Mechanism of Nanoscale Electronic Disorder in $\mathrm{Bi}_{2} \mathrm{Sr}_{2} \mathrm{CaCu}_{2} \mathrm{O}_{8+\delta}$, Science 309, 1048 (2005).

[45] Sen Zhou, Hong Ding, and Ziqiang Wang, Correlating OffStoichiometric Doping and Nanoscale Electronic Inhomogeneity in the High- $T_{c}$ Superconductor $\mathrm{Bi}_{2} \mathrm{Sr}_{2} \mathrm{CaCu}_{2} \mathrm{O}_{8+\delta}$, Phys. Rev. Lett. 98, 076401 (2007).

[46] I. Zeljkovic, Z. Xu, J. Wen, G. Gu, R. S. Markiewicz, and J. E. Hoffman, Imaging the Impact of Single Oxygen Atoms on Superconducting $\mathrm{Bi}_{2+y} \mathrm{Sr}_{2-y} \mathrm{CaCu}_{2} \mathrm{O}_{8+x}$, Science 337, 320 (2012).

[47] Liang Ren Niestemski and Ziqiang Wang, Valence Bond Glass Theory of Electronic Disorder and the Pseudogap State of High-Temperature Cuprate Superconductors, Phys. Rev. Lett. 102, 107001 (2009). 
[48] We average over 40 disorder realizations on samples of $20 \times 20$ sites. To reduce the finite-size effects, for each disorder realization, we average over different boundary conditions corresponding to $10 \times 10$ supercells.

[49] L. K. Werake and H. Zhao, Observation of SecondHarmonic Generation Induced by Pure Spin Currents, Nat. Phys. 6, 875 (2010).

[50] J. Li et al., Direct Detection of Pure ac Spin Current by X-Ray Pump-Probe Measurements, Phys. Rev. Lett. 117, 076602 (2016).

[51] J. C. Wang et al., Decoupling of the Antiferromagnetic and Insulating States in Tb-Doped $\mathrm{Sr}_{2} \mathrm{IrO}_{4}$, Phys. Rev. B 92, 214411 (2015).

[52] L. Zhang, F. Wang, and D.-H. Lee, Compass Impurity Model of Tb Substitution in $\mathrm{Sr}_{2} \mathrm{IrO}_{4}$, Phys. Rev. B 94, 161118(R) (2016).

[53] Feng Ye, Songxue Chi, Bryan C. Chakoumakos, Jaime A. Fernandez-Baca, Tongfei Qi, and G. Cao, Magnetic and Crystal Structures of $\mathrm{Sr}_{2} \mathrm{IrO}_{4}$ : A Neutron Diffraction Study, Phys. Rev. B 87, 140406(R) (2013).

[54] Chetan Dhital, Tom Hogan, Z. Yamani, Clarina de la Cruz, Xiang Chen, Sovit Khadka, Zhensong Ren, and Stephen D. Wilson, Neutron Scattering Study of Correlated
Phase Behavior in $\mathrm{Sr}_{2} \mathrm{IrO}_{4}$, Phys. Rev. B 87, 144405 (2013).

[55] S. Boseggia, R. Springell, H. C. Walker, H. M. Rønnow, Ch. Rüegg, H. Okabe, M. Isobe, R. S. Perry, S. P. Collins, and D. F. McMorrow, Robustness of Basal-Plane Antiferromagnetic Order and the $J_{\text {eff }}=1 / 2$ State in Single-Layer Iridate Spin-Orbit Mott Insulators, Phys. Rev. Lett. 110, 117207 (2013).

[56] D. H. Torchinsky, H. Chu, L. Zhao, N. B. Perkins, Y. Sizyuk, T. Qi, G. Cao, and D. Hsieh, Structural Distortion-Induced Magnetoelastic Locking in $\mathrm{Sr}_{2} \mathrm{IrO}_{4}$ Revealed through Nonlinear Optical Harmonic Generation, Phys. Rev. Lett. 114, 096404 (2015).

[57] C. L. Kane and E. J. Mele, $Z_{2}$ Topological Order and the Quantum Spin Hall Effect, Phys. Rev. Lett. 95, 146802 (2005).

[58] S. D. Matteo and M. R. Norman, Magnetic Ground State of $\mathrm{Sr}_{2} \mathrm{IrO}_{4}$ and Implications for Second-Harmonic Generation, Phys. Rev. B 94, 075148 (2016).

[59] S. Raghu, X.-L. Qi, C. Honerkamp, and S.-C. Zhang, Topological Mott Insulators, Phys. Rev. Lett. 100, 156401 (2008).

[60] S. M. Young and C. L. Kane, Dirac Semimetals in Two Dimensions, Phys. Rev. Lett. 115, 126803 (2015). 